\title{
A IMPLEMENTAÇÃO DA CLASSIFICAÇÃO INTERNACIONAL DE FUNCIONALIDADE INCAPACIDADE E SAÚDE COMO REFERÊNCIA PARA A CLASSIFICAÇÃO DAS NECESSIDADES EDUCATIVAS ESPECIAIS
}

\author{
Anabela Teles ${ }^{1}$ \\ Célia Ribeiro ${ }^{2}$ \\ Cristina Ferreira ${ }^{3}$
}

\begin{abstract}
RESUMO: Para a inclusão de crianças e jovens com Necessidades Educativas Especiais (NEE) no ensino regular foram teorizadas e implementadas práticas com vista a estruturar o Programa Educativo Individual (PEI), adequando-o a cada um, dando respostas à sua singularidade. A implementação do uso da Classificação Internacional de Funcionalidade (CIF), como referência para a classificação das NEE, surge com o Decreto-Lei $n .^{\circ}$ 3/2008, de 7 de janeiro e adota um cariz biopsicossocial criado pela Organização Mundial de Saúde (OMS, 2004).

A sua introdução na educação não tem sido pacífica, sobressaindo o debate entre especialistas e autores que alimentam o esgrimir de argumentos a favor e contra a sua utilização. Analisaremos, assim, a CIF, bem como o seu surgimento, a sua estrutura e as opiniões de alguns especialistas.
\end{abstract}

Palavras-Chave: Inclusão; Necessidades Educativas Especiais; Classificação Internacional de Funcionalidade, Incapacidade e Saúde; Classificação Internacional de Funcionalidade, Incapacidade e Saúde - Crianças e Jovens.

\begin{abstract}
For the Inclusion of children and youth with Special Education Needs (SEN) in regular education, practices were theorized and implemented to structure the Individual Educative Program (IEP), adapting it to each one, responding to their uniqueness. The implementation of the use of the International Classification of Functioning (ICF) as a classification arises with the Decree Law N. ${ }^{\circ}$
\end{abstract}

\footnotetext{
1 anabelateles@gmail.com

2 cribeiro@crb.ucp.pt

3 cristina-ferreira@iol.pt
} 
3/2008, from January 7 and it adopts a biopsicosocial nature created by the World Health Organization (WHO, 2004).

Its introduction in education hasn't been peaceful, exceeding the debate between specialists and authors that fed the arguments in favor and against its use. We'll analyze the ICF, as well as its appearance, structure and the opinions of some specialists.

Keywords: Inclusion; Special Education Needs; International Classification of Functioning, Disabilities and Health; International Classification of Functioning for Children and Youth.

\section{INTRODUÇÃO}

A Educação Especial (EE) em Portugal tem evoluído, refletindo a produção legislativa, os acordos e estudos levados a cabo não só a nível internacional como a nível nacional.

Decorrente da imposição legal da aplicação do Decreto-Lei n. ${ }^{\circ}$ 3/2008, de 7 de janeiro, foi introduzida a utilização do quadro referencial da Classificação Internacional de Funcionalidade, Incapacidade e Saúdeversão para Crianças e Jovens (CIF-CJ). Esta classificação é um complemento à CIF, dado que contempla a especificidade do desenvolvimento na faixa etária compreendida entre o nascimento e os 17 anos de idade. Neste contexto, a avaliação por referência à CIF (funções e estruturas do corpo) e CIF-CJ (atividades e participação e fatores ambientais), segundo a legislação em vigor, permite a elegibilidade dos alunos com NEE de carácter permanente.

Assim, analisamos a origem desta classificação bem como os aspetos estruturais que a compõem, apresentando algumas das opiniões que se têm destacado na sua análise.

\section{CONCEITO E ORIGEM DA CLASSIFICAÇÃO INTERNACIONAL DE FUNCIONALIDADE, INCAPACIDADE E SAÚDE PARA CRIANÇAS E JOVENS}

A CIF, International Classification of Functioning, Disabilities and Health (ICF), é um novo sistema de classificação incluído na Família de Classificações Internacionais da Organização Mundial de Saúde (OMS), que foi aprovada em maio de 2001, por 191 países, como um quadro de referência internacional para descrever e medir a saúde e a incapacidade quer ao nível individual, quer ao nível da população, visando a sua utilização nos diferentes países associados (World Health Organization, 2001). 
Sendo a CIF, frequentemente, referenciada como uma classificação dos estados de saúde e estados relacionados com a saúde, importa reter a conceção presente neste documento, como um "conceito muito abrangente que se prende com os diferentes sectores da vida, com a funcionalidade humana, com o bem-estar e com a qualidade de vida da pessoa" (SEAR, SNRIPD, 2006, p. 16).

A aplicação generalizada da CIF no âmbito da EE condicionada pela ausência de uma versão para crianças e jovens foi, numa fase inicial, uma das principais desvantagens. Em outubro de 2007, em Veneza, a OMS lança a International Classification of Functioning Children and Youth (ICF-CY). Em Língua Portuguesa, existe uma versão experimental traduzida e adaptada com base na CIF (2003), elaborada pelo Centro de Psicologia do Desenvolvimento e da Educação da Criança, da Faculdade de Psicologia e de Ciências da Educação da Universidade do Porto, da qual constam somente as suas componentes: atividades e participação e fatores ambientais.

A CIF-CJ, embora obedecendo à estrutura e organização da CIF original, tem a intenção de facilitar a continuidade na identificação da funcionalidade, atividades e participação e o papel que o ambiente desempenha nas alterações do ser humano, desde a infância até à idade adulta (Sousa, 2007a). Visa, igualmente, facilitar a comunicação entre profissionais, prestadores de serviços e pais, englobando um total de 237 novos códigos que contemplam especificidades da infância e adolescência, com especial relevo para questões do desenvolvimento e crescimento, tais como: a criança no contexto da família; o atraso de desenvolvimento; a participação e os contextos da criança.

Atendendo a que a CIF-CJ tem importância no âmbito do ciclo da vida, a mesma pode ser usada na educação "como um referencial para analisar a funcionalidade, monitorizar progressos e planear as intervenções" (Sousa, 2007a, p. 81).

À luz de um modelo biopsicossocial, Ferreira (2008, citada por DGIDC, 2008a) considera que a CIF-CJ "é mais do que um mero somatório das informações provenientes das várias disciplinas" (p. 17). Faculta aos profissionais intervenientes "no processo de avaliação e de intervenção elaborarem uma visão holística da criança e jovem em contexto e, dessa forma, contribuírem para o desenho das intervenções que mais otimizem os fatores de funcionalidade e perspetivem a participação numa ótica inclusiva" (p. 17).

Através desta visão holística, a CIF-CJ "revela-se como um instrumento unificador que proporciona um trabalho interdisciplinar, promovendo, portanto, práticas de cooperação entre serviços" (Sousa, 2008, citado por DGIDC, 2008a, p. 19), tornando-a, num "canal de acesso 
a apoios coordenados, relacionados com as necessidades funcionais das crianças e jovens" (p. 19). Por outro lado, Gaia (2008, citado por DGIDC, 2008a) defende que a CIF-CJ tem "como mérito o tentar normalizar conceitos usando uma linguagem comum" (p. 21).

\section{ASPETOS ESTRUTURAIS DA CLASSIFICAÇÃO INTERNACIONAL DE FUNCIONALIDADE, INCAPACIDADE E SAÚDE PARA CRIANÇAS E JOVENS}

A CIF encontra-se organizada em duas partes, cada uma delas com duas componentes. Segundo a OMS (2004), a parte 1 diz respeito à funcionalidade e incapacidade e inclui: funções e estruturas do corpo e atividades e participação. A parte 2 trata dos fatores contextuais, incluindo: fatores ambientais e fatores pessoais (atualmente não classificados na CIF, dado que são descritivos e respeitam a individualidade de cada um).

No intuito de homogeneizar conceitos, importa explicar os que a CIF padroniza, que se prendem com as quatro componentes que a integram: funções do corpo; atividades e participação; fatores ambientais e fatores pessoais (OMS, 2004).

O termo incapacidade (disability), introduzido pela CIF, é genérico e utilizado para deficiência, limitação das atividades e restrição na participação. É resultante da interação entre a disfunção apresentada pelo indivíduo (orgânica e/ou da estrutura do corpo), a limitação das suas atividades e a restrição social, bem como os fatores ambientais que podem atuar como facilitadores ou barreiras no desempenho dessas atividades e da participação (Farias \& Buchalla, 2005). Por sua vez, o termo deficiência, reportando-se ao estado da pessoa, não é compatível com a CIF, uma vez que esta se refere somente às alterações ou anomalias ao nível das estruturas e funções do corpo, sem que daí se possa estabelecer uma relação causal para a sua funcionalidade/incapacidade (Vale, 2009).

A funcionalidade é o termo genérico para as funções e estruturas do corpo, atividades e participação social. Corresponde aos aspetos positivos da interação entre um indivíduo (com uma condição de saúde) e os seus fatores contextuais. Assim se conclui que, na CIF, a funcionalidade é usada como aspeto positivo e a incapacidade corresponde ao aspeto negativo (Farias \& Buchalla, 2005).

Cada componente da CIF contém vários capítulos e domínios. Em cada um, há várias categorias e subcategorias que constituem as unidades de classificação. Os qualificadores são códigos numéricos que especificam a extensão ou magnitude da funcionalidade numa 
determinada categoria e em que medida um fator ambiental constitui um facilitador ou uma barreira.

Estes componentes são identificados por prefixos em cada código e utiliza o seguinte sistema alfa-numérico: b (body) para funções do corpo; $\mathrm{s}$ (structure) para estruturas do corpo; d (domain) para atividades e participação; e (environment) para fatores ambientais (cf. Quadro I).

Neste sentido, "todos os componentes são quantificados através da mesma escala genérica. Ter um problema pode significar uma deficiência, limitação, restrição ou barreira, dependendo do constructo" ${ }^{4 \prime}$ (OMS, 2004, p. 196).

As funções do corpo são funções fisiológicas dos sistemas orgânicos, incluindo as funções psicológicas (cf. Quadro II).

\section{Quadro I}

Códigos do sistema alfanumérico

\begin{tabular}{|l|l|}
\cline { 2 - 2 } \multicolumn{1}{l|}{} & \multicolumn{1}{c|}{ Exemplo: } \\
\hline $\begin{array}{l}1^{\circ}{ }^{\circ} \text { Código numérico (um } \\
\text { dígito) - capítulo }\end{array}$ & $\begin{array}{l}\text { Capítulo 1 - Funções Mentais (Funções Mentais } \\
\text { Específicas) }\end{array}$ \\
\hline $\begin{array}{l}2 .^{\circ} \text { Código numérico (dois } \\
\text { dígitos) - categoria }\end{array}$ & b140 - Funções da Atenção \\
\hline $\begin{array}{l}3 .^{\circ} \text { Código numérico (um } \\
\text { dígito cada) }\end{array}$ & b1400 - Manutenção da Atenção \\
\hline
\end{tabular}

Fonte: OMS (2004)

\section{Quadro II}

Funções e estruturas do corpo

\begin{tabular}{|l|l|}
\hline Capítulo 1 & Funções mentais \\
\hline Capítulo 2 & Funções sensoriais e dor \\
\hline Capítulo 3 & Funções da voz e da fala \\
\hline Capítulo 4 & $\begin{array}{l}\text { Funções do aparelho cardiovascular, dos sistemas hematológico e } \\
\text { imunológico e do aparelho respiratório }\end{array}$ \\
\hline Capítulo 5 & $\begin{array}{l}\text { Funções do aparelho digestivo e dos sistemas metabólico e } \\
\text { endócrino }\end{array}$ \\
\hline Capítulo 6 & Funções genitourinárias e reprodutivas \\
\hline Capítulo 7 & $\begin{array}{l}\text { Funções neuromusculoesquelécticas e funções relacionadas com o } \\
\text { movimento }\end{array}$ \\
\hline Capítulo 8 & Funções da pele e estruturas relacionadas \\
\hline
\end{tabular}

Fonte: OMS (2004)

\footnotetext{
${ }^{4}$ Constructos: são definidos através do uso de qualificadores com códigos relevantes. Há quatro constructos para a Parte 1: Mudanças nas Funções do Corpo - "orgânicas"; Mudanças na Estrutura do Corpo - "anatómicas"; Capacidade; Desempenho; e, um para a Parte 2: Facilitadores ou Barreiras em Fatores Ambientais (OMS, 2004).
} 
As deficiências são problemas nas funções ou estruturas do corpo, tais como um desvio ou perda significativos. Podem ser temporárias ou permanentes; progressivas, regressivas ou estáveis; intermitentes ou contínuas (OMS, 2004).

De acordo com a CIF (OMS, 2004), as categorias da componente funções do corpo são quantificadas com um qualificador que indica a extensão ou magnitude de deficiência, de acordo com a escala apresentada (cf. Quadro III).

\section{Quadro III}

Categorias da componente funções do corpo

\begin{tabular}{|l|l|c|}
\hline xxx.0 & NENHUMA deficiência (Nenhuma, ausente, escassa...) & $0-4 \%$ \\
\hline xxx. 1 & Deficiência LIGEIRA (leve, nenhuma, ...) & $5-24 \%$ \\
\hline xxx.2 & Deficiência MODERADA (média, ...) & $25-49 \%$ \\
\hline $\mathbf{x x x . 3}$ & Deficiência GRAVE (grande, extrema, ...) & $50-95 \%$ \\
\hline $\mathbf{x x x . 4}$ & Deficiência COMPLETA (total, ...) & $96-100 \%$ \\
\hline $\mathbf{x x x . 8}$ & não especificada & - \\
\hline xxx.9 & não aplicável & - \\
\hline $\begin{array}{l}\text { NOTA: As percentagens devem ser calibradas, tendo como referência, os valores } \\
\text { standards da população como percentis. }\end{array}$
\end{tabular}

Fonte: OMS (2004)

As atividades são a execução de tarefas ou ações por um indivíduo e a participação é o envolvimento do indivíduo numa situação de vida real. De acordo com a OMS (2004), limitações das atividades são dificuldades que o indivíduo pode ter na execução de atividades e restrições de participação são problemas que a pessoa pode enfrentar quando está envolvida em situações da vida real. Esta componente está dividida em nove capítulos (cf. Quadro IV).

\section{Quadro IV}

Atividades e participação

\begin{tabular}{|l|l|}
\hline Capítulo 1 & Aprendizagem e aplicação de conhecimentos \\
\hline Capítulo 2 & Tarefas e exigências gerais \\
\hline Capítulo 3 & Comunicação \\
\hline Capítulo 4 & Mobilidade \\
\hline Capítulo 5 & Auto cuidados \\
\hline Capítulo 6 & Vida doméstica \\
\hline Capítulo 7 & Interações e relacionamentos interpessoais \\
\hline Capítulo 8 & Áreas principais de vida \\
\hline Capítulo 9 & Vida comunitária, social e cívica \\
\hline
\end{tabular}

Fonte: OMS (2004) 
As categorias da componente atividades e participação são quantificadas através da escala que a seguir se apresenta (cf. Quadro V).

\section{Quadro V}

Categorias da componente atividades e participação

\begin{tabular}{|l|l|c|}
\hline $\mathbf{x x x . 0}$ & NENHUMA dificuldade (Nenhuma, ausente, escassa...) & $0-4 \%$ \\
\hline $\mathbf{x x x . 1}$ & Dificuldade LIGEIRA (leve, nenhuma, ...) & $5-24 \%$ \\
\hline $\mathbf{x x x . 2}$ & Dificuldade MODERADA (média, ...) & $25-49 \%$ \\
\hline $\mathbf{x x x . 3}$ & Dificuldade GRAVE (grande, extrema, ...) & $50-95 \%$ \\
\hline $\mathbf{x x x . 4}$ & Dificuldade COMPLETA (total, ...) & $96-100 \%$ \\
\hline $\mathbf{x x x . 8}$ & não especificada & - \\
\hline $\mathbf{x x x . 9}$ & não aplicável & - \\
\hline
\end{tabular}

Fonte: OMS (2004)

Definem-se como fatores ambientais os que "constituem o ambiente físico, social e atitudinal em que as pessoas vivem e conduzem a sua vida" (OMS, 2004, p. 152). Estes fatores são externos ao indivíduo e podem ter uma influência positiva ou negativa sobre o seu desempenho enquanto membro da sociedade, ou sobre as funções ou estruturas do corpo (cf. Figura I).

A CIF considera facilitadores os fatores ambientais que, através da sua ausência ou presença, melhoram a funcionalidade e reduzem a incapacidade de uma pessoa. Estes fatores incluem aspetos como um ambiente físico que seja acessível, disponibilidade de tecnologia apropriada, atitudes positivas das pessoas em relação à incapacidade, bem como serviços, sistemas e políticas que tendem a aumentar o envolvimento de todas as pessoas com uma condição de saúde em todas as áreas de vida (OMS, 2004).

Por outro lado, as barreiras são fatores ambientais que, através da sua ausência ou presença, limitam a funcionalidade e provocam a incapacidade. Estes fatores incluem aspetos como um ambiente físico inacessível, falta de tecnologia de assistência apropriada, atitudes negativas das pessoas em relação à incapacidade, bem como os serviços, sistemas e políticas inexistentes ou que dificultam o envolvimento de todas as pessoas com uma condição de saúde em todas as áreas de vida (OMS, 2004).

Esta componente encontra-se dividida em cinco capítulos com as denominações que se seguem (cf. Figura II). 


\section{Figura I}

Coeficientes dos fatores ambientais

Fonte: OMS (2004)

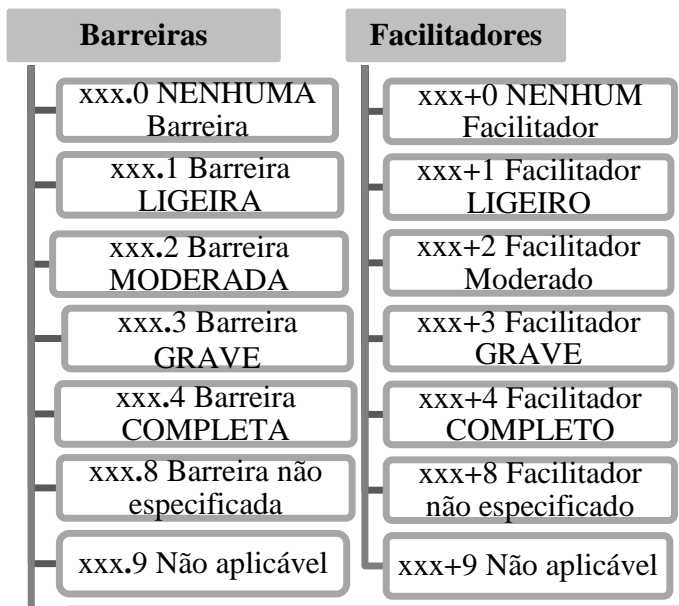

Nota: Um coeficiente ( 0 a 4 ) separado por um ponto (.) indica uma barreira. Se estiver separado do código pelo sinal (+) indica um facilitador.

Figura II

Fatores ambientais

Capítulo 1 - Produtos e tecnologia

Capítulo 2 - Ambiente natural e mudanças ambientais feitas pelo homem

Capítulo 3 - Apoios e relacionamentos

Capítulo 4 - Atitudes

Capítulo 5 - Serviços, sistemas e políticas

Fonte: OMS (2004) 
Com referência à componente fatores pessoais, a DGIDC (2006) menciona que os mesmos não são classificados na CIF, embora os utilizadores os possam incorporar nas suas aplicações da classificação. Estes fatores são o histórico particular da vida e do estilo de vida de um indivíduo e englobam as suas características, que não são parte de uma condição de saúde ou de uma condição relacionada com a saúde. Podem incluir o sexo, idade, outros estados de saúde, condição física, estilo de vida, hábitos, educação recebida, diferentes maneiras de enfrentar problemas, antecedentes sociais, nível de instrução, profissão, experiência passada e presente, padrão geral de comportamento, carácter, características psicológicas individuais e outras características, todas ou algumas das quais podem desempenhar um papel na incapacidade em qualquer nível.

\section{A CLASSIFICAÇÃO INTERNACIONAL DE FUNCIONALIDADE, INCAPACIDADE E SAÚDE COMO MUDANÇA DE PARADIGMA}

Com a aprovação do novo sistema de classificação, CIF, introduz-se uma mudança radical de paradigma, passando do modelo puramente médico/individual, para um modelo biopsicossocial e completado pela funcionalidade e incapacidade humana, sintetizando estes dois modelos numa visão coerente das diferentes perspetivas de saúde: biológica, individual e social (OMS, 2004).

Ferreira (2008) lembra que o novo paradigma precisava de "um processo de avaliação e de registo que traduzisse as diferentes componentes do desenvolvimento e de uma linguagem descritiva transversal aos diferentes técnicos" (p. 7). Ainda segundo esta autora "a CIF responde a estas necessidades, permitindo passar do absoluto da deficiência ao relativismo da incapacidade" (p. 8).

Sustentando o novo paradigma, Capucha (2009) considera a CIF como um modelo consequente ao combinar o modelo médico e o modelo social. $\mathrm{Na}$ sua opinião, a CIF constitui uma "classificação universal do funcionamento humano" (p. 14). Assim, segundo o autor, o modelo "não classifica os indivíduos de modo essencialista, mas sim em função dos contextos em que operam as capacidades e incapacidades, permitindo ou não a participação" (p. 15).

Perante a afirmação da OMS de que a CIF vai permitir "uma linguagem unificada e padronizada, bem como uma estrutura de trabalho comum para a descrição da saúde e de estados relacionados com a saúde" (DGIDC, 2006, p. 14), Kauffman e Simpson (2007) consideram que esta 
conceção é médica, embora inclua fatores ambientais, capacidades, funcionalidades e incapacidades do sujeito. $\mathrm{O}$ citado autor confirma que "a adoção de uma classificação de cariz médico representa uma significativa alteração na conceção das sinalizações para o ensino especial e representa um retorno a um modelo médico da deficiência" (p. 63). Este modelo, desde a publicação do Decreto-Lei n. ${ }^{\circ}$ 319/1991, de 23 de agosto, já tinha sido substituído por um modelo psicopedagógico.

Em análise ao tema, Correia e Lavrador (2010) antecipam que "o melhor modelo de incapacidade é aquele que sintetiza o que existe de verdadeiro nos modelos médico e social, sem incorrer no erro de reduzir a noção global e complexa de incapacidade apenas a um dos seus aspetos" (p. 16). Sendo o modelo CIF biopsicossocial, integra os modelos médico e social, constituindo assim, um modelo de incapacidade mais promissor.

A CIF é uma ferramenta a utilizar universalmente na abordagem da incapacidade e funcionalidade humana, proporcionando-nos: um quadro concetual de referência universal assente em bases científicas; uma linguagem comum e padronizada para aplicação universal que uniformiza conceitos e terminologias, de modo a facilitar a comunicação entre profissionais, investigadores, pessoas com incapacidades, decisores, políticos, etc.; um sistema de classificação multidimensional e de codificação sistemática para documentar as experiências de vida, o perfil funcional e de participação dos indivíduos, facilitando a comparabilidade entre países, entre várias disciplinas, entre serviços e em diferentes momentos ao longo do tempo (INR, 2010). A sua aplicação, pode igualmente ser efetuada de forma transversal, em diferentes áreas disciplinares e setores: da saúde, da educação, da segurança social, do emprego, da economia e desenvolvimento, das estatísticas e sistemas de informação e legislação.

Em defesa desta conceção, a ativista internacional das deficiências e incapacidades, que participou no desenvolvimento da CIF, Hurst (2001, citada por Sousa, 2007a) menciona que esta ferramenta, "se usada adequadamente, suporta o modelo dos direitos relativo às deficiências e incapacidades e irá ajudar-nos a colher as provas que mostram como a nossa vida realmente é" (p. 67). Refere ainda que a incapacidade está relacionada com o "impacto que os fatores ambientais têm na deficiência e funcionalidade" (p. 67).

Segundo Vale (2009), para além da sua aplicação diversificada, tem igualmente múltiplas finalidades nas atuações e intervenções relacionadas com a incapacidade: a nível clínico/individual (avaliação individual do indivíduo, planeamento das intervenções, reabilitação, etc.); a nível institucional (planeamento e avaliação de serviços e recursos, formação dos profissionais, investigação, etc.); e a nível social e político 
(planeamento, desenvolvimento e avaliação de políticas e medidas; sistemas de compensação e de atribuição de benefícios; critérios de elegibilidade; acessibilidade; indicadores e estatísticas, etc.).

Nas palavras de Sousa (2007a), a CIF "oferece uma descrição híbrida e útil de deficiências e incapacidades" (p. 66), que pode ser usada por todos, com a finalidade de chegarem a um consenso, relativamente ao significado de deficiências e incapacidades, facultando "um referencial abrangente para operacionalizar essa descrição" (p. 66).

\section{A CLASSIFICAÇÃO INTERNACIONAL DE FUNCIONALIDADE, INCAPACIDADE E SAÚDE COMO UM REFERENCIAL PARA A AVALIAÇÃO}

Esta classificação "não é um instrumento de avaliação nem de medida, mas sim um quadro de referência e um conjunto de classificações, nos quais os instrumentos de avaliação e de medida se devem basear e estar relacionados" (WHO-FIC, 2008, p. 7), contudo, "não dispensa que os profissionais, dentro das suas áreas de especialidade, adotem procedimentos e utilizem instrumentos de avaliação normalizados e fidedignos que evidenciem de forma rigorosa os diferentes domínios em estudo, tomando como referência a CIF" (INR, 2010, s/p).

Para efetuar o processo de avaliação em EE é necessária uma equipa multidisciplinar para apoiar as necessidades específicas de cada criança/jovem, numa ótica de rentabilização de recursos (docentes do ensino regular, dos serviços especializados de apoio educativo, um elemento da família do aluno, profissionais de projetos de parceria, profissionais das equipas de saúde escolar, etc.). No âmbito da efetivação da avaliação, é necessário selecionar as categorias que, em cada componente, irão ser objeto de classificação, tendo em conta as categorias constantes na checklist e a condição específica de cada criança/jovem.

Dispondo da informação existente sobre o aluno, bem como da que foi recolhida pelos diferentes técnicos, torna-se necessário proceder à análise conjunta desta mesma informação, com o objetivo de se definir o perfil de funcionalidade do aluno em questão. Este é composto por duas componentes que entre si se complementam: uma de carácter mais descritivo e que nos dá o perfil intraindividual e, outra, de carácter mais normativo, que permite determinar o seu perfil interindividual (DGIDC, 2006).

Por último, surge a tomada de decisões para efeitos da planificação da intervenção educativa. Para a planificação do processo de recolha de 
informação, é elaborado um documento, intitulado Roteiro de Avaliação. Após análise conjunta dos dados da avaliação, é elaborado um relatório técnico-pedagógico, que deverá ainda explicar as razões determinantes das NEE e a sua tipologia, bem como as respostas e medidas educativas a adotar e que servirão de base à elaboração do PEI.

$\mathrm{O}$ PEI é um documento formal que garante o direito à equidade educativa e que responsabiliza os intervenientes pela implementação de medidas educativas que promovam a aprendizagem e a participação dos alunos com NEE de carácter permanente; é um instrumento de trabalho que descreve o perfil de funcionalidade por referência à CIF-CJ e estabelece as respetivas respostas educativas específicas; é um instrumento dinâmico que deve ser revisto e reformulado, pois assenta numa avaliação compreensiva e integrada do funcionamento do aluno, suscetível de sofrer alterações (DGIDC, 2008b).

\section{DIVERGÊNCIAS NA UTILIZAÇÃO DA CLASSIFICAÇÃO INTERNACIONAL DE FUNCIONALIDADE, INCAPACIDADE E SAÚDE}

A natureza e aplicabilidade desta classificação tem provocado divergências de opinião dos diversos autores e especialistas neste domínio, que vão desde a sua génese à sua aplicação, levando à tomada de posições com argumentos a favor e contra.

Das vozes que levantam argumentos contra a utilização desta classificação, destaca-se Correia (2008), que afirma que o Decreto-Lei n. ${ }^{\circ}$ $3 / 2008$, de 7 de janeiro é restritivo e discriminatório, no sentido em que "parece excluir a maioria dos alunos com NEE permanentes, deixando de fora mais de $90 \%$ desses alunos" (p. 73). Este autor refere que "a DGIDC comete mais um erro de palmatória ao pretender identificar e classificar estes alunos" (Correia, 2007, p. 1) por referência à CIF-CJ. O autor acrescenta que "não é difícil perceber-se que a CIF emana de uma instituição especializada das Nações Unidas, eminentemente clínica, a Organização Mundial de Saúde" (p. 1). Refere, ainda, que sendo a CIF uma classificação que diz respeito à saúde, a maioria dos profissionais nem sequer a usa e o facto da educação a utilizar pode trazer consequências desastrosas para os alunos com NEE.

Também Lopes (2007) considera que "as linguagens de médicos e psicólogos por um lado e de professores por outro, são porventura demasiado clivadas e vincadas para que haja um significativo entendimento mútuo" (p. 63). 
Na opinião dos membros do Fórum de Estudos de Educação Inclusiva (FEEI, s/d), o uso de uma classificação de funcionalidade provinda da saúde para fins educacionais, pressupõem "um grave erro" (p. 1).

Relativamente à aplicabilidade da CIF, e no que concerne ao seu instrumento de recolha de dados de informação da CIF-CJ, Correia (2008) alerta que a mesma "é uma rating scale (escala gradativa) e não uma checklist (lista de verificação) " (p. 75). Ao ser utilizada como instrumento informal de recolha de informação, tratando-se de uma rating scale, é referido pelo autor como um "procedimento muito confuso para todos aqueles que tenham de graduar comportamentos" (p. 75), originando o mesmo caso interpretações diferentes.

Sousa (2007b) refere que especialistas e Governo convergem num aspeto, "assumem a importância de se elaborar uma só peça legislativa, que dê suporte a um modelo de educação especial" ( $\mathrm{s} / \mathrm{p}$ ).

Por outro lado, temos os defensores do modelo CIF, desde logo Simeonsson (2009) que considera que "a CIF não exclui ou inclui" (p. 1). Baseia-se na avaliação de funções e capacidade, de acordo com os contextos envolventes, permitindo obter respostas adequadas a cada caso. A aplicação deste quadro referencial vai assegurar o seu direito aos serviços sociais, educativos, de apoio e de saúde, prevenindo a discriminação.

Hollenweger (2008, citado por DGIDC, 2008b) refere três pontos principais para a utilização da CIF-CJ. O primeiro prende-se com o facto de esta classificação poder ser usada como uma ferramenta concetual para compreender as necessidades especiais no contexto educativo, uma vez que as suas categorias ajudam a identificar os problemas das crianças e jovens que nem sempre se relacionam com a saúde, permitindo assim a interação entre a pessoa e o ambiente que a envolve. Em segundo lugar, permite organizar a planificação e a tomada de decisão dos profissionais, pela partilha de informação comum e por criar uma moldura que define a experiência profissional e a escolha de instrumentos de apoio. Por último, ajuda a organizar processos de elegibilidade para apoio da EE ou para a utilização de recursos adicionais nos sistemas educativos, permitindo cruzar informação e indicadores para desenvolver políticas que possam ser traduzidas numa intervenção adequada.

Perspetivando a deficiência e ou doença como uma experiência universal, Vale (2009) considera que a CIF vem facilitar a comunicação e o trabalho dos técnicos envolvidos ao criar uma linguagem uniforme e acessível e ao enfatizar a importância da interação entre a criança e o meio, tendo em conta o seu bem-estar biopsicossocial.

Ferreira (2009a) defende que "a Classificação Internacional de Funcionalidade (CIF) tem atributos que lhe permitem instituir-se como 
instrumento passível de pragmatizar a intervenção e o pensamento subjacente ao movimento inclusivo, através de um conhecimento e de uma linguagem unificadora" (s/p). Para que tal aconteça, a utilização da CIF não é suficiente. Os profissionais terão que ir para além dos seus campos específicos de trabalho para apreender a totalidade do indivíduo como uma "entidade dinâmica e holisticamente organizada" (s/p).

Pelas inúmeras dúvidas surgidas quanto ao seu preenchimento (checklist - funções do corpo), Palha (2009) esclarece que a CIF “é particularmente útil em crianças ou adolescentes com Deficiência Cognitiva ou perturbações similares (por exemplo, gravíssimas alterações comportamentais, ...), não se adaptando bem às Perturbações Específicas do Desenvolvimento, uma vez que não se baseia na teoria e métodos do Desenvolvimento Infantil' (p. 1).

Recentemente, Simeonsson e colaboradores (2010) reafirmam que a CIF não é unicamente um sistema classificador das dimensões da saúde, do funcionamento e das atividades e participação das crianças e jovens, uma vez que estes são atributos e experiências destas crianças e jovens inseridas em determinadas situações e circunstâncias. Assim sendo, para justificar a aplicação dos códigos utilizados pela CIF devem ser efetuadas medidas com base em testes e instrumentos independentes.

\section{CONCLUSÃO}

A CIF apresenta-se como um sistema de classificação universal aprovado pela OMS, em 2001, constituindo-se como um referencial internacional que proporciona um quadro concetual de referência e uma linguagem comum de descrição e medição da saúde e da incapacidade de um indivíduo ou de uma população. Como sistema de classificação reveste-se de um carácter abrangente considerando os aspetos da funcionalidade humana, do bem-estar e da qualidade de vida da pessoa. A CIF-CJ surge como uma derivação da primeira, adaptada para crianças e jovens.

A introdução da CIF traz consigo uma mudança de paradigma, passando-se de um modelo médico para um modelo biopsicossocial, onde se encontram integradas a funcionalidade e a incapacidade. A aplicação da CIF no processo de avaliação das NEE preconiza uma linguagem unificada bem como uma estrutura de trabalho comum, interativa e multidimensional, no âmbito de um trabalho de equipa transdisciplinar.

Sousa (2007a) refere a CIF como modelo operativo de caracterização da funcionalidade do indivíduo, em conformidade com o modelo social. O autor corrobora a ideia afirmando que o mais importante não é a avaliação das alterações ao nível das estruturas ou funções do indivíduo, 
"mas sim, as limitações da atividade e as restrições da participação que o sujeito experiencia no decorrer da interação entre as suas caraterísticas biopsicológicas e as caraterísticas dos ambientes sociais em que se move" (p. 50).

Autores como Breia e Micaelo (2008) e Ferreira (2009b), consideram positivo o maior envolvimento e responsabilização dos intervenientes no processo de avaliação com a constituição das equipas multidisciplinares. Candeias e colaboradores (2009) referem nas suas conclusões a ideia de que a linguagem da CIF-CJ é unificadora e padronizada de forma a facilitar o trabalho dos diversos intervenientes no processo de avaliação. Também Vale (2009) nos apresenta esta ideia de que a CIF possibilita uma linguagem específica que a torna acessível aos mais diversos técnicos e Profissionais da Saúde e da Educação que estão ligados ao processo de avaliação de crianças/jovens, ajudando assim a ultrapassar as habituais dificuldades não só de comunicação como de articulação entre os intervenientes neste processo.

Como quadro referencial, constitui-se como um importante instrumento de avaliação utilizando a checklist (funções do corpo, atividades e participação, e fatores ambientais e fatores pessoais) para a elaboração de um relatório técnico-pedagógico onde se identifica o perfil de funcionalidade do aluno, servindo de base à elaboração do PEI.

As opiniões dos diversos autores e investigadores, utilizando argumentos a favor e contra, tem permitido perspetivar a aplicabilidade deste sistema de classificação no processo de avaliação das NEE.

Para os diversos Profissionais e Técnicos que trabalham com crianças e jovens com vista à sua inclusão familiar, escolar, social e/ou profissional, cabe a tarefa de, como refere Vale (2009), "esclarecer a sociedade, sensibilizá-la nas vertentes humanista, ética e utilitarista" (p. 236), colocando à disposição das crianças e jovens com NEE e respetivas famílias todos os meios e recursos com vista às suas necessidades acrescidas.

\section{REFERÊNCIAS BIBLIOGRÁFICAS}

Breia, G., \& Micaelo, M. (2008). Relatório final - Acompanhamento e monitorização do processo de aplicação do referencial proposto pela CIF. Açores: Direcção Regional de Educação dos Açores.

Candeias, A., Rosário, A., Saragoça, M., Rebocho, M., Pastor, G., Coincas, J., Cortes, M., \& Rocha, O. (2009). Desafios à avaliação e intervenção educativa: Reflexões sobre a experiência de implementação da CIF em Portugal. Centro de Investigação em Educação e Psicologia. Évora: Universidade de Évora. 
Capucha, L. (2009). Inovação e justiça: Políticas activas para a inclusão educativa. Seminário Internacional Educação Inclusiva - Impacto dos Referenciais Internacionais nas Políticas, nas Práticas e na Formação. Lisboa: DGIDC.

Correia, L. (2007). Carta aberta à Senhora Ministra da Educação. Educare.pt. Consultado em 24 de Agosto de 2010, de http://www.educare.pt/educare/Opiniao.Artigo.aspx?contentid=AD8 AC917C7D14D529E51EBD93B5432EE\&opsel $=2 \&$ channelid $=0$

Correia, L. (2008). A Escola contemporânea e a inclusão de alunos com NEE - Considerações para uma Educação com Sucesso. Porto: Porto Editora.

Correia, L., \& Lavrador, R. (2010). A utilização da CIF em educação: um estudo exploratório. EE/DPEEE. Braga: Instituto de Educação (IE). Universidade do Minho.

DGIDC (2006). Avaliação e intervenção na área das NEE. Consultado em 12 de Janeiro de 2010, de http://www.appdae.net/documentos/manuais/avaliacao.pdf

DGIDC (2008a). Encontro temático - Educação especial. Programa. Notas curriculares. Resumo das comunicações. Lisboa: Ministério da Educação.

DGIDC (2008b). Educação especial - Manual de apoio à prática. Lisboa: Ministério da Educação.

Farias, N., \& Buchalla, C. (2005). A classificação internacional de funcionalidade, incapacidade e saúde da Organização Mundial da Saúde: Conceitos, usos e perspectivas. Revista Brasileira de Epidemiologia. Faculdade de Saúde Pública de São Paulo, 8(2), 187193.

FEEI (s/d). Tomada de posição do FEEI sobre a utilização da CIF como "Paradigma na avaliação das NEE". Faculdade de Motricidade Humana. Consultado a 05 de Setembro de 2010, de http://www.spmram.org/conteudo/ficheiros/legislacao/especial/FEEI-CIF.pdf

Ferreira, M. (2008). A formação dos profissionais para a utilização da CIF-CJ. Encontro temático de Educação Especial. Consultado em 10 de Agosto de 2010, de http://area.dgidc.minedu.pt/pdf_especial/formacaoCIF_CJ.pdf

Ferreira, M. (2009a). O modelo biopsicossocial na formação dos profissionais na utilização da CIF - Crianças e jovens. Consultado em 12 de Fevereiro de 2010, de

http://srec.azores.gov.pt/Encontro_Tematico/Convidados/1Conferencia.ht $\mathrm{m}$ 
Ferreira, M. (Coord.) (2009b). Projecto de avaliação da implementação do Decreto-Lei $n^{\circ}$. 3/2008: Resultados preliminares. Consultado em 04 de Junho de 2010, de http://www.dgidc.minedu.pt/especial/Documents/Manuela\%20Sanches\%20Ferreira2.pdf

INR (2010). Como surgiu a CIF?. Consultado em 20 de Maio de 2010, de http://www.inr.pt/content/1/55/que-cif.

Kauffman, J., \& Simpson, R. (2007). Inclusão de alunos deficientes em sala de aula regulares. In J. Kauffman \& J. Lopes (Coord.). Pode a educação especial deixar de ser especial? (pp. 167-185). Braga: Psiquilíbrios Edições.

Lopes, J. (2007). Perspectiva crítica da educação especial em Portugal. In J. Kauffman \& J. Lopes (Coord.). Pode a educação especial deixar de ser especial? (pp. 21-94). Braga: Psiquilíbrios Edições.

OMS (2004). Classificação Internacional de Funcionalidade, Incapacidade e Saúde - CIF. Genebra: Autor.

Palha, M. (2009). CIF. Centro de Desenvolvimento Infantil Diferenças. Consultado em 05 de Fevereiro de 2010, de http://diferencas.net/cif.html.

SEAR, SNRIPD (2006). $1^{\circ}$ Plano de Acção para a Integração das Pessoas com Deficiências ou Incapacidades. Lisboa: Ministério do Trabalho e da Solidariedade Social.

Simeonsson, R. (2009). Educação especial: Sistema de sinalização sob avaliação. Consultado em 18 de Outubro de 2010, de http://www.educare.pt/educare/Detail.aspx?contentid=5AC8628C0C 97E59FE0400A0AB8002122\&opsel=1\&schema=1CD970AB08363 34EB627B1FF128684C3\&channelid=1EE474ED3B3E054C8DCFD 48A24FF0E1B

Simeonsson, R., Tavares, A., Pinheiro, S., Ferreira, M., Maia, M., \& Alves, S. (2010). Síntese da apresentação dos resultados da avaliação externa da implementação do Decreto-Lei $n^{o}$ 3/2008. Consultado em 30 de Julho de 2010, de http://sitio.dgidc.minedu.pt/especial/Documents/S\%C3\%ADnteseAp resPublicaResultados.pdf

Sousa, J. (Coord.) (2007a). Mais qualidade de vida para as pessoas com deficiências e incapacidades - Uma estratégia para Portugal. Vila Nova de Gaia: Centro de Reabilitação Profissional de Gaia (CRPG) e Instituto Superior de Ciências do Trabalho e da Empresa (ISCTE).

Sousa, T. (2007b). Especialistas contra o método CIF. Consultado em 28 de Dezembro de 2009, de http://www.educare.pt/educare/Detail.aspx?contentid=DDFE97165F 524553A8EF8742039E6821\&opsel=1\&schema=1CD970AB083633 
4EB627B1FF128684C3\&channelid=1EE474ED3B3E054C8DCFD4 8A24FF0E1B.

Vale, M. (2009). Classificação Internacional de Funcionalidade (CIF): Conceitos, preconceitos e paradigmas. Contributo de um constructo para o percurso real em meio natural de vida. Acta Pediátrica Portuguesa. Sociedade Portuguesa de Pediatria. (Setembro/Outubro 2009).

World Health Organization (2001). International Classification of Functioning, Disability and Health (ICF). Geneva.

WHO-FIC Network (World Health Organization Family of International Classifications); WG (2008). Briding Task Group (BTG). Draft report for annual WHO-FIC meeting. Outubro. Consultado em 26 de Fevereiro de 2010, de http://www.nsbc.gov.ph/events/disability/papers/Day2/Draft\%20Mi nutes $\% 20$ of $\% 20$ Meeting.doc.

\section{REFERÊNCIAS NORMATIVAS}

Decreto-Lei n. ${ }^{\circ} 319 / 1991$, de 23 de agosto

Decreto-Lei n. ${ }^{\circ}$ 3/2008, de 7 de janeiro 